\section{BRAZIULIAN JOURNAL \\ OF MEDICAL AND BIOLOGICAL RESEARCH}

www.bjournal.com.br
ISSN 1414-431X

Volume 45 (12) 1102-1340 December 2012

\section{BIOMIDICAL SCIENCES}

AND

CLINICAL INVESTIGATION

Braz J Med Biol Res, December 2012, Volume 45(12) 1334-1340

doi: 10.1590/S0100-879X2012007500163

\title{
Clinicopathological significance of PTPN12 expression in human breast cancer
}

Yuan Xunyi, Yuan Zhentao, Jiang Dandan and Li Funian

The Brazilian Journal of Medical and Biological Research is partially financed by

\section{욛NPq}

da Ciência e Tecnologia

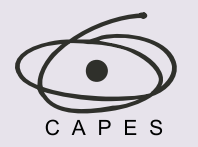

Ministério da Educação

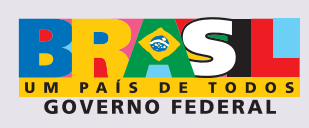

DTFAPESP

Institutional Sponsors

Ф SHIMADZU

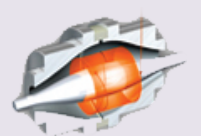

Sciefo
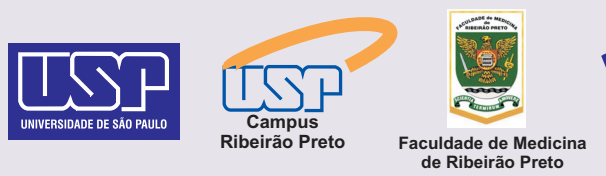

UNICAMP

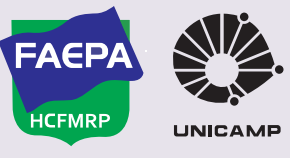

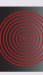
de Incentivo
a Pesquisa

Explore High - Performance MS Orbitrap Technology

\section{analitica Thermo}




\title{
Clinicopathological significance of PTPN12 expression in human breast cancer
}

\author{
Yuan Xunyi ${ }^{1 *}$, Yuan Zhentao ${ }^{2 *}$, Jiang Dandan ${ }^{1}$ and Li Funian ${ }^{1 *}$ \\ ${ }^{1}$ Breast Disease Diagnosis and Treatment Centre, Affiliated Hospital of Medical College, \\ Qingdao University, Qingdao, Shandong Province, China \\ ${ }^{2}$ Department of Anesthesiology, Shengli Oilfield Central Hospital, Dongying, Shandong Province, China
}

\begin{abstract}
Protein tyrosine phosphatase non-receptor type 12 (PTPN12) is a recently identified tumor suppressor gene (TSG) that is frequently compromised in human triple-negative breast cancer. In the present study, we investigated the expression of PTPN12 protein by patients with breast cancer in a Chinese population and the relationship between PTPN12 expression levels and patient clinicopathological features and prognosis. Additionally, we explored the underlying down-regulation mechanism from the perspective of an epigenetic alteration. We examined PTPN12 mRNA expression in five breast cancer cell lines using semiquantitative reverse-transcription PCR, and detected PTPN12 protein expression using immunohistochemistry in 150 primary invasive breast cancer cases and paired adjacent non-tumor tissues. Methylation-specific PCR was performed to analyze the promoter CpG island methylation status of PTPN12. PTPN12 was significantly down-regulated in breast cancer cases (48/150) compared to adjacent noncancerous tissues (17/150; $\mathrm{P}<0.05)$. Furthermore, low expression of PTPN12 showed a significant positive correlation with tumor size $(P=0.047)$, lymph node metastasis $(P=0.001)$, distant metastasis $(P=0.009)$, histological grade $(P=0.012)$, and survival time $(P=0.019)$. Additionally, promoter $C p G$ island hypermethylation occurs more frequently in breast cancer cases and breast cancer cell lines with low PTPN12 expression. Our findings suggest that PTPN12 is potentially a methylation-silenced TSG for breast cancer that may play an important role in breast carcinogenesis and could potentially serve as an independent prognostic factor for invasive breast cancer patients.
\end{abstract}

Key words: Breast cancer; Tumor suppressor gene; PTPN12; Prognosis; Methylation

\section{Introduction}

Approximately one million new cases of breast cancer are diagnosed each year worldwide and it is the most common neoplastic disease in women (1). Triple-negative breast cancer (TNBC), characterized by a lack of ER, $\mathrm{PR}$, and HER2 expression, accounts for approximately $15 \sim 20 \%$ of the cases of all breast cancer types (2). TNBC is an aggressive malignancy that exhibits a poor prognosis and whose treatment is limited and often ineffective. The molecular mechanisms underlying TNBC development are not clear.

PTPN12, located in 7q11.23, is a member of the protein tyrosine phosphatase (PTP) family. Accumulating evidence indicates that there is a correlation between PTPN12 and tumor development, including ovarian cancer, colon cancer, and prostate cancer (3-5). Recent reports have demonstrated that PTPN12 inactivation leads to HER2/EGFR hyperactivity and cellular transformation in HER2-negative breast cancer and that PTPN12 is frequently compromised in human TNBC, indicating that it is potentially a new tumor suppressor gene (TSG) of TNBC (6).

The mechanisms underlying TSG inactivation primarily include two aspects: genetic change and epigenetic change. Aberrant methylation of promoter $\mathrm{CpG}$ islands is one of the most important epigenetic mechanisms that causes TSG silencing and leads to transcription repressor binding, compressed chromatin, and transcription silencing in the early stages of tumor genesis $(7,8)$. Currently, research concerning the mechanisms underlying PTPN12 inactivation has been limited to genetic changes and, to our knowledge, there are no reports to date concerning PTPN12 gene promoter methylation. In the present study, we hypothesized that the silencing of PTPN12 could be caused by methylation.

In this study, our findings indicated that PTPN12 is a

Correspondence: Li Funian, Breast Disease Diagnosis and Treatment Centre, Affiliated Hospital of Medical College, Qingdao University, Qingdao, Shandong Province, 266003, China. E-mail: yzhtyxy@163.com

*These authors contributed equally to this study.

Received July 7, 2012. Accepted August 29, 2012. Available online October 15, 2012. Published December $17,2012$. 
potential TSG for breast cancer and a candidate that may serve as a prognostic factor in breast cancer. The present study is the first to examine the expression profile of PTPN12 in a comparatively large group of Chinese breast cancer patients with sufficient follow-up data, and the first to analyze the association of PTPN12 expression with clinicopathological and prognostic characteristics. Furthermore, we investigated whether promoter $\mathrm{CpG}$ island methylation is correlated with PTPN12 inactivation mechanisms using methylation-specific PCR (MSP).

\section{Material and Methods}

\section{Cell lines and tumor samples}

Five human breast cancer cell lines (HCC1937, MDAMB-231, MCF-7, BT-474, and SK-BR-3) were purchased from the China Center for Type Culture Collection, Chinese Academy of Sciences. HCC1937 and MDA-MB-231 are basal-like subtype, MCF-7 is luminal A subtype, BT-474 is luminal B subtype, and SK-BR-3 is HER2 subtype. The cells were routinely maintained in RPMI or DMEM medium with $10 \%$ fetal bovine serum (Hyclone, USA) at $37^{\circ} \mathrm{C}$ in a humidified atmosphere of $5 \% \mathrm{CO}_{2}$. Formalin-fixed, paraffin-embedded samples of 150 primary invasive breast cancer tissues and paired adjacent non-tumor tissues were obtained from the Qingdao University Affiliated Hospital from January to December 1999. Our research was approved by the Institutional Ethics Committee of Qingdao University Affiliated Hospital and informed written consent was obtained from all patients.

\section{Cases and clinical data}

The median age of the patients was 50.5 years (range: 29-87 years). The duration of follow-up ranged from 2 to 134 months (median: 85.7 months); all patients underwent a follow-up of over 36 months unless they died of any cause. Cases lost to follow-up were excluded from the study. Pathological diagnoses were routinely assessed by pathologists in the Pathology Department, Qingdao University Affiliated Hospital. None of the patients had received prior radiotherapy or neo-adjuvant therapy. All patients received conventional postoperative treatment, depending on the extent of disease. Patients without axillary lymph node involvement were treated with surgical treatment alone, whereas patients with axillary lymph node involvement received six courses of adjuvant chemotherapy with a cyclophosphamide/methotrexate/fluorouracil regimen. Patients with positive nodes or tumor size $\geq 5 \mathrm{~cm}$ received postoperative radiation. Patients with $E R+/ P R+$ tumors were treated for 2-5 years with tamoxifen.

\section{RNA extraction and semi-quantitative reverse- transcription PCR}

Total RNA was extracted from each sample using Trizol (Invitrogen, USA) and reverse transcribed into cDNA us- ing the PrimeScript RT-PCR kit (TaKaRa Bio Inc., Japan) according to manufacturer recommendations. The primers for the human PTPN12 gene were designed with Primer Premier 5.0 (Premier Biosoft, USA) and the sequences were sense: 5'-AAATACTGCAGCCACCGGAAC-3', antisense: 5'-GCAACACTGGCTTTGGATGG-3'; the amplicon size was $126 \mathrm{bp}$. GAPDH was used as the internal control with the specific primers - sense: 5'-TCATGGGTGTGAACCATGAGAA-3', antisense: 5'-GGCATGGACTGTGGTCATGAG-3'; the amplicon size was $150 \mathrm{bp}$. The primers cited above were synthesized by Shanghai Sangon Biological Engineering Technology \& Services Co. Ltd. (China). The PCR products were analyzed by $1 \%$ agarose gel electrophoresis.

\section{Immunohistochemistry}

Expression levels of HER2, ER, PR, and PTPN12 were investigated in 150 primary breast cancer tissue specimens and paired adjacent non-tumor tissues using immunohistochemistry. Antibodies to ER, PR, and HER2 were purchased from ZSGB-BIO (ZSGB, China). Rabbit polyclonal antibody to PTPN12 was purchased from Abcam (Abcam plc, UK). The PTPN12 protein was detected primarily in the cytoplasm. ER and PR were positive primarily in the cell nucleus, whereas HER2 was detected in the cell membrane and cytoplasm.

To determine PTPN12 levels, each sample was assigned to 5 categories and the proportion of positive-stained cells was rated as follows: 0 (0-4\%), 1 (5-24\%), 2 (25-49\%), 3 $(50-74 \%)$, or $4(75-100 \%)$. The intensity of immunostaining was categorized as 0 (no stain), $1+$ (weak stain), 2+ (medium stain), and $3+$ (strong stain). In addition, an immunoreactive score was calculated by multiplying the percentage of positive cells and the stain intensity. In instances of heterogeneous staining intensity within one sample, each component was scored independently and the results were summed. For statistical analysis, the criteria were combined as follows: $0=$ negative; 1 to $4=$ weakly positive; 5 to $8=$ moderately positive, and 9 to $12=$ strongly positive (9). In the present study, low expression was defined as negative and weakly positive, and high expression was defined as moderately positive and strongly positive. The Allred scoring system (10) was used for ER and PR staining interpretation and HER2 was evaluated according to ASCO guidelines (11).

\section{Methylation-specific PCR}

Genomic DNA was extracted from cells and tissues using standard phenol-chloroform extraction. EpiScope Methylated HeLa gDNA (TaKaRa Bio Inc.) was used as a positive control for methylation analysis. Placenta tissue gDNA was also extracted and used as a negative control. Bisulfite modification of DNA was produced using the Zymo DNA Modification-Gold Kit (Zymo Research, USA) according to manufacturer recommendations. The bisulfite-treated DNA was amplified with methylation and 
unmethylation primers, which were designed with Methyl Primer Express v1.0 (ABI, USA). Methylation primer sense: 5'-TCGTTTGTGAAGAAGGTATTTC-3', antisense: 5'-AAAAAACGAAACGCTTCCTA-3'. Unmethylation primer - sense: 5'-GTTGTTTGTGAAGAAGGTATTTT-3', antisense: 5'-AAAAAAACAAAACACTTCCTA-3'. The PCR product sizes were both $104 \mathrm{bp}$. Methylation-specific PCR for the PTPN12 promoter was conducted in a total PCR volume of $20 \mu \mathrm{L}$. MSP primers were tested previously to ensure that no unbisulfited DNA was amplified and to confirm that our MSP system is specific. The PCR products were analyzed by $1 \%$ agarose gel electrophoresis.

\section{Statistical analysis}

Statistical analyses were performed using the SPSS statistical software (SPSS, Inc., USA). The correlations between PTPN12 expression and clinicopathological variables were analyzed using Pearson chi-square analysis. The same method was used to test for associations of PTPN12 with ER, PR, and HER2. Kaplan-Meier survival analysis and univariate analysis of the differences between the survival curves were tested using the log-rank test. Uni- variate and multivariate survival analyses were performed using Cox proportional hazards regression. A value of $\mathrm{P}<$ 0.05 indicated a statistically significant result.

\section{Results}

PTPN12 mRNAtranscription was down-regulated in one of the triple-negative breast cancer cell lines (HCC1937) as well as in two other types of cell lines (MCF-7, BT-474; Figure 1). PTPN12 protein immunohistochemistry (Figure 2) demonstrated that PTPN12 protein expression was down-regulated (negative and weak expression) in $32.0 \%$ of tumor specimens $(48 / 150)$ and $11.3 \%$ of paired adjacent non-tumor tissues (17/150), with a significant difference $(P$ $=0.00009)$. The PTPN12 protein exhibited low expression in $34.7 \%$ of TNBC specimens $(26 / 75)$ and $29.3 \%$ of nonTNBC specimens $(22 / 75)$ with no significant difference $(P$ $=0.556$; Table 1$)$.

As shown in Table 1, significant positive correlations were found between low-level expression of the PTPN12 protein and tumor size $(P=0.047)$, lymph node metastasis $(P=0.001)$, histological grade $(P=0.012)$, and distant

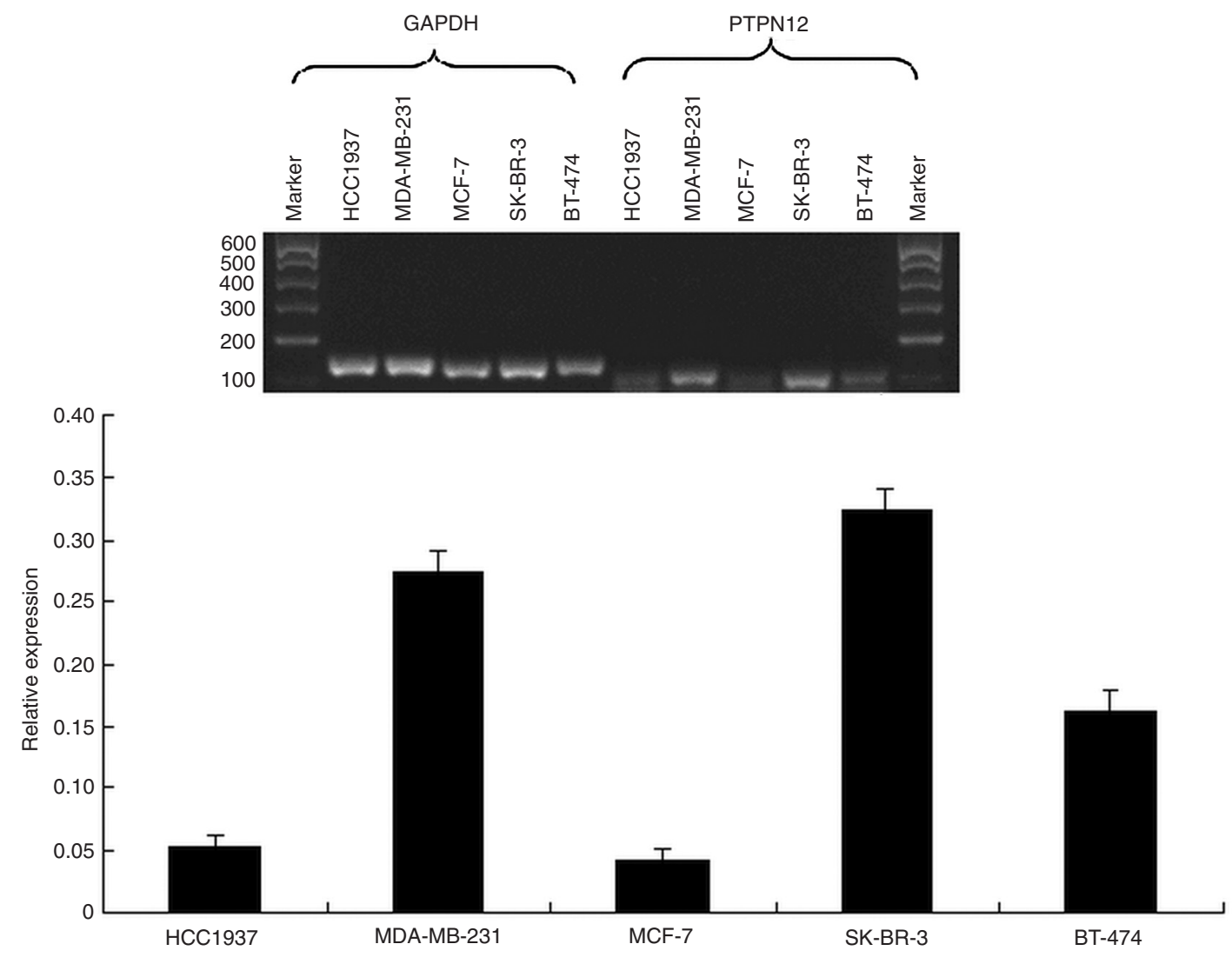

Figure 1. Expression of protein tyrosine phosphatase non-receptor type 12 (PTPN12) mRNA in five breast cancer cell lines detected by RT-PCR. PTPN12 transcription was down-regulated in HCC1937, MCF-7, and BT-474. GAPDH = glyceraldehyde-3-phosphate dehydrogenase. 


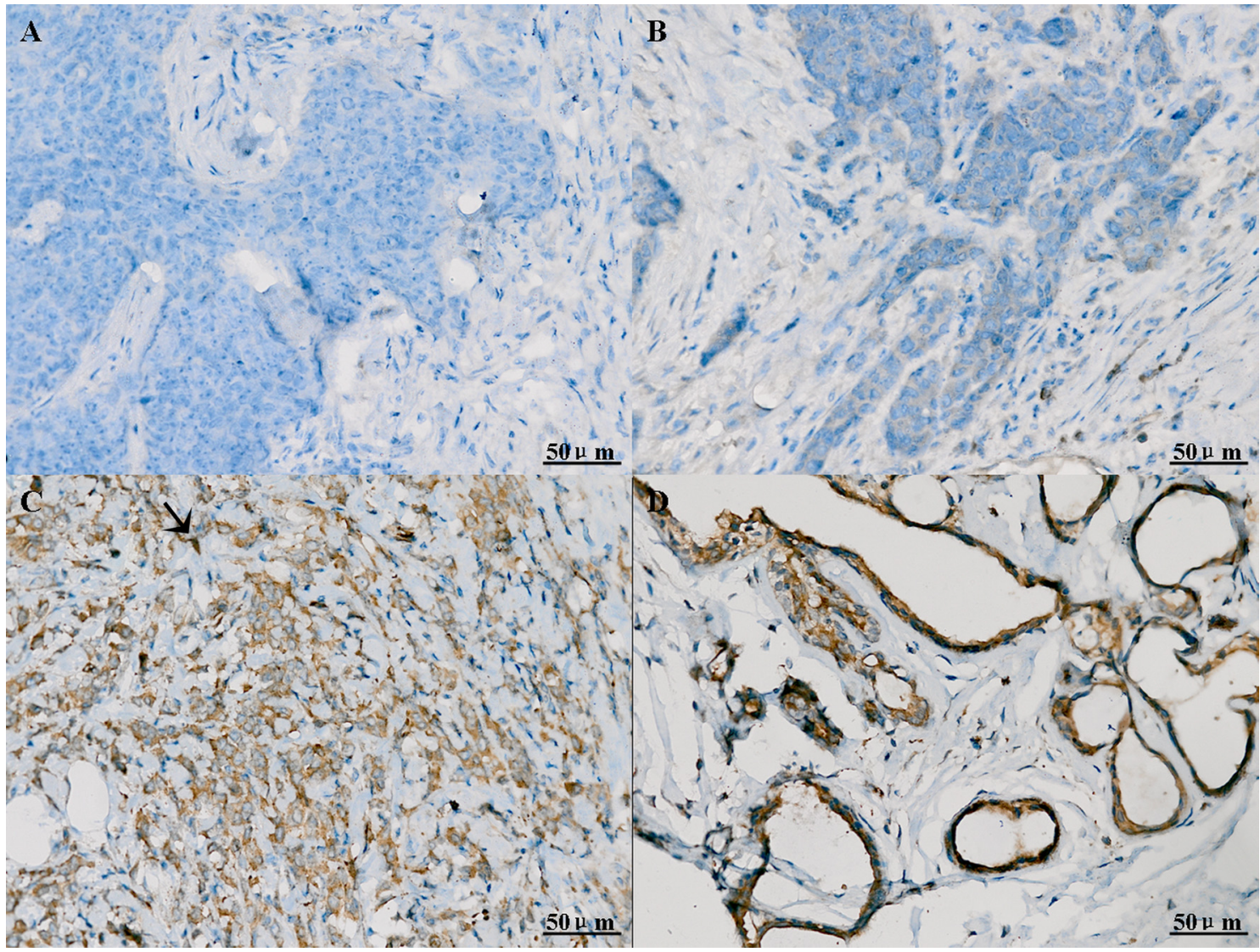

Figure 2. Representative immunohistochemistry of protein tyrosine phosphatase non-receptor type 12 (PTPN12) in breast cancer tissues. $A$, Negative. $B$, Weakly positive (+). $C$, High expression of PTPN12 in tumor (++ to +++). $D$, High expression of PTPN12 in adjacent tissues. $A-D$, PTPN12 protein was detected primarily in the cytoplasm. The brown staining (arrow) indicates positive staining, and the blue color is the counterstain of the nuclei.

metastasis $(P=0.009)$. However, there was no significant correlation between PTPN12 expression and age, and no significant correlation between PTPN12 expression and cancer recurrence (local/regional) by statistical analysis. There was no statistically significant difference between PTPN12 expression levels and HER2 or EGFR (P > 0.05).

Kaplan-Meier analysis demonstrated significant differences in overall survival between patients with high-level PTPN12 protein expression and patients with low-level PTPN12 expression. Moreover, low-level PTPN12 expression was associated with decreased overall survival $(P=$ 0.019; Figure 3). Cox univariate survival analysis demonstrated that tumor size, lymph node status, histological grade, and PTPN12 expression levels were statistically significant risk factors that affected the outcome of patients with breast cancer. In multivariate survival analysis, lymph node status, histological grade, tumor size, and PTPN12 expression were independent prognostic factors (Table 2).

Methylation was observed in two of three PTPN12 down-regulated cell lines (MCF-7 and BT-474; Figure 4). We then examined PTPN12 promoter methylation in 150 formalin-fixed paraffin-embedded primary breast cancer tumor specimens and their paired adjacent non-tumor tissues using MSP. A total of 39.6\% PTPN12 low protein expression specimens (19/48) exhibited methylation (Figure 4) whereas none exhibited methylation in the PTPN12 high protein expression specimens and non-tumor specimens $(P<0.05)$.

\section{Discussion}

PTPN12 is among the PTPs that regulate the equilibrium of tyrosine phosphorylation and play a prominent role in tumor suppression. Loss of PTPN12 leads to malignant transformation of human mammary epithelial cells, and restoration of PTPN12 expression in PTPN12-deficient breast cancer cells inhibits their proliferation, tumorigenicity, and metastatic potential (6). The present study demonstrates for the first time the relationship between the characteristics of PTPN12 expression and patient prognosis in human breast cancer and investigated the mechanisms underlying down-regulation of PTPN12 expression through the 
Table 1. Association of protein tyrosine phosphatase non-receptor type 12 (PTPN12) expression with clinicopathological parameters.

\begin{tabular}{|c|c|c|c|c|c|}
\hline \multirow[t]{2}{*}{ Characteristics } & \multirow[t]{2}{*}{$\mathrm{N}$} & \multicolumn{2}{|c|}{ PTPN12 } & \multirow[t]{2}{*}{$\mathrm{P}$} & \multirow[t]{2}{*}{$x^{2}$} \\
\hline & & Low expression & High expression & & \\
\hline \multicolumn{6}{|l|}{ Cases } \\
\hline Tumor tissues & 150 & $48(32.00)$ & $102(68.00)$ & 0.000 & 18.88 \\
\hline Non-tumor tissues & 150 & $17(11.33)$ & $133(88.67)$ & & \\
\hline \multicolumn{6}{|l|}{ Age (years) } \\
\hline$\leq 50$ & 66 & $26(39.39)$ & $40(60.61)$ & 0.085 & 2.961 \\
\hline$>50$ & 84 & $22(39.47)$ & $62(60.53)$ & & \\
\hline \multicolumn{6}{|l|}{ Tumor size } \\
\hline$\leq 2 \mathrm{~cm}$ & 74 & $18(24.32)$ & $56(75.68)$ & 0.047 & 3.954 \\
\hline$>2 \mathrm{~cm}$ & 76 & $30(39.47)$ & $46(60.53)$ & & \\
\hline \multicolumn{6}{|c|}{ Lymph node metastasis } \\
\hline Positive & 57 & $32(56.14)$ & $25(43.86)$ & 0.001 & 11.84 \\
\hline Negative & 93 & $26(27.96)$ & $67(72.04)$ & & \\
\hline \multicolumn{6}{|l|}{ Distant metastasis } \\
\hline Present & 31 & $16(51.60)$ & $15(48.40)$ & 0.009 & 6.908 \\
\hline Absent & 119 & $32(26.90)$ & $87(73.10)$ & & \\
\hline \multicolumn{6}{|c|}{ Recurrence (local/regional) } \\
\hline Present & 24 & $7(29.20)$ & $17(70.8)$ & 0.745 & 0.105 \\
\hline Absent & 126 & $41(32.5)$ & $85(67.5)$ & & \\
\hline \multicolumn{6}{|l|}{ Histological grade } \\
\hline 1 & 16 & $4(25.00)$ & $12(75.00)$ & 0.012 & 8.884 \\
\hline II & 65 & $12(33.85)$ & $43(66.15)$ & & \\
\hline III & 69 & $32(46.38)$ & $37(53.62)$ & & \\
\hline \multicolumn{6}{|l|}{ Molecular subtype } \\
\hline Luminal A & 36 & $10(27.78)$ & $22(61.12)$ & 0.311 & 3.575 \\
\hline Luminal B & 18 & $3(16.67)$ & $15(83.33)$ & & \\
\hline HER2 & 20 & $9(45.00)$ & $11(55.00)$ & & \\
\hline Basal (TNBC) & 76 & $26(34.21)$ & $50(65.79)$ & & \\
\hline \multicolumn{6}{|l|}{ Luminal A subtype } \\
\hline Yes & 36 & $10(27.78)$ & $26(72.22)$ & 0.814 & 0.055 \\
\hline No & 114 & $34(29.82)$ & $80(70.18)$ & & \\
\hline \multicolumn{6}{|l|}{ Luminal B subtype } \\
\hline Yes & 18 & $3(16.67)$ & $15(83.33)$ & 0.137 & 2.210 \\
\hline No & 132 & $45(34.09)$ & 87 (65.91) & & \\
\hline \multicolumn{6}{|l|}{ HER2 subtype } \\
\hline Yes & 20 & $9(45.00)$ & $11(55.00)$ & 0.276 & 1.189 \\
\hline No & 130 & $39(32.23)$ & $81(67.77)$ & & \\
\hline \multicolumn{6}{|l|}{ Basal subtype } \\
\hline Yes & 76 & $26(34.21)$ & $50(76.92)$ & 0.556 & 0.346 \\
\hline No & 74 & $22(29.73)$ & $52(70.27)$ & & \\
\hline
\end{tabular}

Data are reported as number with percent in parentheses. TNBC = triple-negative breast cancer.

examination of epigenetic alterations.

Sun et al. (6) reported that PTPN12 was undetectable in $37 \%$ of invasive breast cancer cases, $9.1 \%$ of HER2amplified tumors, and $60.4 \%$ of TNBC cases. These findings indicate that PTPN12 is a new tumor suppressor gene for TNBC. In the present study, down-regulation of PTPN12 mRNA was detected in three of five cell lines by RT-PCR and not only in TNBC. To further elucidate the correlations of PTPN12 expression levels in breast cancer tissues, we investigated PTPN12 protein expression in 


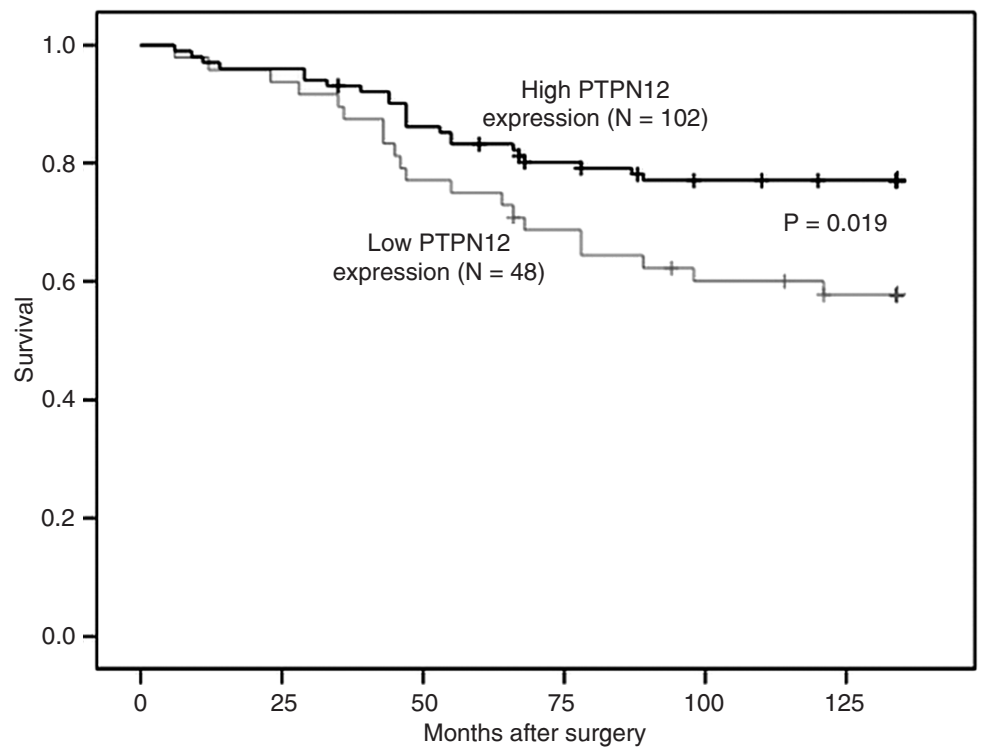

Figure 3. Correlation between protein tyrosine phosphatase non-receptor type 12 (PTPN12) expression and cumulative survival rate in breast cancer patients, as determined by the Kaplan-Meier method. The survival of patients with a low expression of PTPN12 was worse than that of patients with high PTPN12 expression $(P=0.019)$.
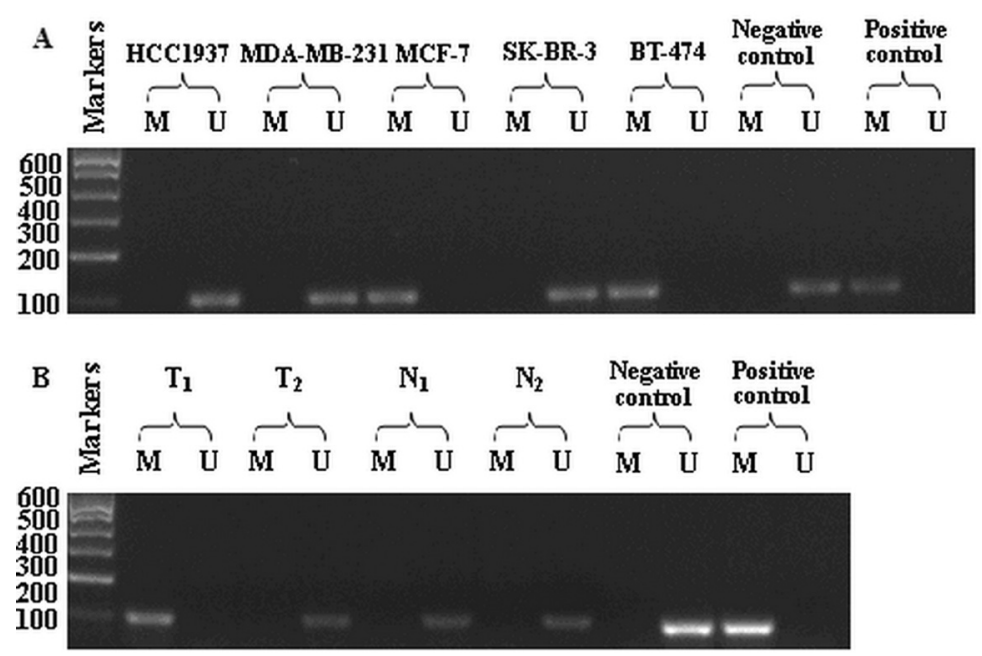

Figure 4. Analysis of protein tyrosine phosphatase non-receptor type 12 (PTPN12) methylation by methylation-specific PCR (MSP). A, MSP results of five breast cancer cell lines. $B$, MSP results of breast cancer samples $\left(T_{1-2}\right)$ and their matched normal renal tissues $\left(\mathrm{N}_{1-2}\right)$. Lanes $M=\mathrm{MSP}$ for methylated promoter; lanes $U=$ MSP for unmethylated promoter.

150 breast cancer cases. Our findings indicated that PTPN12 protein expression decreased in $32.0 \%$ cases of breast cancer and only in $11.3 \%$ paired adjacent non-tumor tissues. Our study confirms that PTPN12 is an important suppressor in breast cancer. Nevertheless, no statistically significant differences were observed here between TNBC and non-TNBC,
Table 2. Univariate and multivariate Cox survival analysis.

\begin{tabular}{lc}
\hline Variable & $\mathrm{P}$ \\
\hline Univariate & \\
Age & 0.472 \\
Tumor size & 0.017 \\
Lymph node status & 0.025 \\
Histological grade & 0.001 \\
PTPN12 expression & 0.020 \\
Multivariate & \\
Tumor size & 0.022 \\
Lymph node status & 0.034 \\
Histological grade & 0.003 \\
PTPN12 expression & 0.022 \\
\hline
\end{tabular}

PTPN12 = protein tyrosine phosphatase non-receptor type 12.

HER2-positive or not. The different conclusions drawn from these results may be due to the variety of racial gene structure and to the experimental methodology. PTPN12 protein expression can be influenced by multiple factors. The underlying regulatory mechanisms remain unclear.

It is understood that the loss of cell-cell adhesion increases invasion and metastasis, which is an important step in carcinoma progression. The PTPN12 protein is thought to act as an important regulator in controlling cell adhesion, motility, and metastasis by interacting with and inhibiting multiple oncogenic tyrosine kinases (12). Silencing of PTPN12 has been shown to enhance migration in ovarian cancer (3) and colon cancer cells (4), and may cause metastasis in breast cancer (6). Analysis of the association of PTPN12 expression with clinicopathological characteristics indicated that low-level PTPN12 expression was strongly associated with worse prognosis, including increased tumor size, histological stage progression, and appearance of lymph node and/or distant metastasis. However, other clinicopathological characteristics such as age and local or regional recurrence exhibited no difference between low- and high-level PTPN12 protein expression. Low PTPN12 expression was significantly associated with poor survival in patients with breast cancer according to Kaplan-Meier analysis and was a statistically significant independent poor prognostic factor by multivariate Cox survival analysis. Thus, the outcomes after surgery are 
significantly poorer in patients with low PTPN12 expression than in those with high expression, indicating that PTPN12 inactivation may result in aggressive proliferation of tumors and can be used as a key marker for the assessment of prognosis in breast cancer.

Interactions of genetic and epigenetic alterations play an important role in tumor genesis. Promoter methylation that causes tumor suppressor gene inactivation is an important epigenetic phenomenon. Aberrant DNA methylation occurs frequently in human breast cancer, is associated with the loss of expression of numerous tumor-suppressor genes, and is correlated with clinical outcomes (13). We observed that promoter $\mathrm{CpG}$ island hypermethylation occurred much more frequently in cell lines or specimens with low PTPN12 expression, indicating that it is potentially an important mechanism underlying PTPN12 down-regulation. We hy-

\section{References}

1. Hoque MO, Prencipe M, Poeta ML, Barbano R, Valori VM, Copetti $\mathrm{M}$, et al. Changes in $\mathrm{CpG}$ islands promoter methylation patterns during ductal breast carcinoma progression. Cancer Epidemiol Biomarkers Prev 2009; 18: 2694-2700.

2. Carey LA, Perou CM, Livasy CA, Dressler LG, Cowan D, Conway $\mathrm{K}$, et al. Race, breast cancer subtypes, and survival in the Carolina Breast Cancer Study. JAMA 2006; 295: 2492-2502.

3. Villa-Moruzzi E. Tyrosine phosphatases in the HER2directed motility of ovarian cancer cells: Involvement of PTPN12, ERK5 and FAK. Anal Cell Pathol 2011; 34: 101-112.

4. Espejo R, Rengifo-Cam W, Schaller MD, Evers BM, Sastry SK. PTP-PEST controls motility, adherens junction assembly, and Rho GTPase activity in colon cancer cells. Am J Physiol Cell Physiol 2010; 299: C454-C463.

5. Sahu SN, Nunez S, Bai G, Gupta A. Interaction of Pyk2 and PTP-PEST with leupaxin in prostate cancer cells. Am J Physiol Cell Physiol 2007; 292: C2288-C2296.

6. Sun T, Aceto N, Meerbrey KL, Kessler JD, Zhou C, Migliaccio $\mathrm{I}$, et al. Activation of multiple proto-oncogenic tyrosine kinases in breast cancer via loss of the PTPN12 phosphatase. Cell 2011; 144: 703-718.

7. Jones PA, Baylin SB. The fundamental role of epigenetic pothesized that demethylation drugs potentially up-regulate PTPN12 expression, which needs to be rigorously tested in further experiments.

Our study confirms that PTPN12 is a tumor suppressor gene of breast cancer. PTPN12 is down-regulated in several breast cancer cell lines and in Chinese primary invasive breast cancer patients. The evidence does not indicate that PTPN12 is a TSG only for TNBC in Chinese people. The present findings are the first to suggest that methylation of the PTPN12 promoter region potentially provides a suitable biomarker for PTPN12 diagnosis and therapy.

\section{Acknowledgments}

Research supported by the Natural Science Foundation of Shandong China Grant (\#Y2007C124). events in cancer. Nat Rev Genet 2002; 3: 415-428.

8. Jones PA, Baylin SB. The epigenomics of cancer. Cell 2007; 128: 683-692.

9. Milde-Langosch K, Bamberger AM, Rieck G, Kelp B, Löning T. Over expression of the p16 cell cycle inhibitor in breast cancer is associated with a more malignant phenotype. Breast Cancer Res Treat 2001; 67: 61-70.

10. Chae BJ, Bae JS, Yim HW, Lee A, Song BJ, Jeon HM, et al. Measurement of ER and PR status in breast cancer using the QuantiGene2.0 assay. Pathology 2011; 43: 248-253.

11. Wolff AC, Hammond ME, Schwartz JN, Hagerty KL, Allred DC, Cote RJ, et al. American Society of Clinical Oncology/ College of American Pathologists guideline recommendations for human epidermal growth factor receptor 2 testing in breast cancer. J Clin Oncol 2007; 25: 118-145.

12. Streit S, Ruhe JE, Knyazev P, Knyazeva T, lacobelli S, Peter $\mathrm{S}$, et al. PTP-PEST phosphatase variations in human cancer. Cancer Genet Cytogenet 2006; 170: 48-53.

13. Feng W, Shen L, Wen S, Rosen DG, Jelinek J, Hu X, et al. Correlation between $\mathrm{CpG}$ methylation profiles and hormone receptor status in breast cancers. Breast Cancer Res 2007; 9: R57. 